\title{
Holomorphic functions of polynomial growth on abelian coverings of a compact complex manifold
}

\author{
Alexander Brudnyi ${ }^{1}$
}

Let $M$ be a compact complex manifold and $p: M_{G} \longrightarrow M$ be a regular covering over $M$ with a free abelian transformation group $G$. We prove under some constraints on $G$ that the space $\mathcal{P}_{l}\left(M_{G}\right)$ of holomorphic functions on $M_{G}$ of $l$-polynomial growth is finite dimensional and is linearly imbedded into the corresponding space $\mathcal{P}_{l}\left(\mathbf{C}^{N}\right)$ of holomorphic polynomials on $\mathbf{C}^{N}$ with $N:=\frac{1}{2} \operatorname{rank}(G)$.

\section{Introduction.}

The classical Liouville theorem asserts that every holomorphic function on $\mathbf{C}^{n}$ of polynomial growth $l$ is a holomorphic polynomial of degree at most $l$. The result has many deep generalizations related to solutions of a linear or non-linear elliptic differential equation on a covering over a Riemannian or complex manifold (see, in particular, $[\mathrm{CM}],[\mathrm{Gu}],[\mathrm{LS}],[\mathrm{K}],[\mathrm{L}],[\mathrm{Li}],[\mathrm{AL}]$, [MS]). In this paper we discuss the problem of the description of the space of holomorphic functions of polynomial growth on a regular covering over a compact complex manifold. To its formulation we henceforth denote by $M$ a compact complex manifold and by $M_{G}$ a regular covering $p: M_{G} \longrightarrow M$ over $M$ with a finitely generated transformation group $G$. Fix a minimal set of generators $e_{1}, \ldots, e_{k}$ of $G$ and introduce the distance $\rho$ on $G$ by

$$
\rho(g, h):=\min \left\{\sum_{i}\left|\alpha_{i}\right| ; g h^{-1}=\prod_{i} e_{i}^{\alpha_{i}}\right\} .
$$

Let $V \subset M_{G}$ be a fundamental compact with respect to the action of $G$, i.e., $M_{G}=\bigcup_{g \in G} g V$.

\footnotetext{
${ }^{1}$ The work was supported in part by the NSERC.
} 
Definition 1.1. A holomorphic function $f$ on $M_{G}$ is said to be of $l$-growth if there exists a constant $C$ such that

$$
\sup _{z \in V}|f(g z)| \leq C \rho(g, 1)^{l} .
$$

The linear space of these functions will be denoted by $\mathcal{P}_{l}\left(M_{G}\right)$.

In the similar way one can introduce the space $\mathcal{H}_{l}\left(M_{G}\right)$ of harmonic functions of $l$-polynomial growth on a regular covering over a compact Riemannian manifold $M$ with transformation group $G$. Recall that a function $f$ on $M_{G}$ is harmonic if it is a solution of the equation $\Delta f=0$, where $\Delta$ is the pullback of the Laplacian on $M$.

The main question is to find out the conditions on $G$ under which the spaces $\mathcal{P}_{l}\left(M_{G}\right)$ ( or $\mathcal{H}_{l}\left(M_{G}\right)$ ) are finite dimensional. In harmonic case the problem is closely related to a conjecture of Yau $[\mathrm{Y}]$ on finite-dimensionality of the space of harmonic functions of polynomial growth defined on a complete Riemannian manifold with non-negative Ricci curvature.

It was firstly proved by Guivarch ([Gu]) and independently by Lyons and Sullivan ([LS]) that in the case of nilpotent $G$ the space $\mathcal{H}_{0}\left(M_{G}\right)$ consists of constants only. Then Kaimanovich $([\mathrm{K}])$ proved that a similar result holds in the case of a polycyclic group $G$. Using a different approach V. Lin ([L]) established that $\mathcal{P}_{0}\left(M_{G}\right)$ consists of constants in the case where $G$ is a nilpotent group. His method gives also a new proof of the LyonsSullivan result. Further, Avallaneda and F.-H. Lin showed that in the case $M_{G}=\mathbf{R}^{n}$ is a covering over a Riemannian manifold $M$ diffeomorphic to a real torus, the space $\mathcal{H}_{l}\left(M_{G}\right)$ is finite dimensional. They gave also an explicit description of the space. A new proof of this result and its generalization to the case of nonlinear elliptic operators see in the paper of Moser and Struve ([MS]).

A. further development in this subject was done at the midst of 1996 due to Colding and Minicozzi. In a series of papers they studied the space of harmonic functions of polynomial growth and eventually proved Yau's conjecture (see, e.g. [CM] for references of all their works). Moreover, their methods allowed them to prove finite-dimensionality of $\mathcal{H}_{l}\left(M_{G}\right)$ for any nilpotent $G$ and give an effective estimate of the dimension. In fact, Colding and Minicozzi proved a more general statement. They considered complete manifolds satisfying the volume doubling property and the Poincaré inequality and proved finite-dimensionality of spaces of harmonic functions of polynomial growth defined on such manifolds. Finally, P. Li ([Li]) gave a deep generalization of the results of Colding and Minicozzy. He established finite-dimensionality of spaces of harmonic functions of polynomial 
growth with better estimates of their dimensions under rather general assumptions on a manifold. Namely, he assumed only the fulfilment of a mean value inequality for nonnegative subharmonic functions and a weak volume comparison condition.

The results described lead naturally to the following conjecture for holomorphic functions of polynomial growth posed by V.Lin.

Let $G$ be a nilpotent group and $M$ be a compact complex manifold. Then $\mathcal{P}_{l}\left(M_{G}\right)$ is finite dimensional. Moreover, every $f \in \mathcal{P}_{l}\left(M_{G}\right)$ (resp., $\mathcal{H}_{l}\left(M_{G}\right)$ ) is annihilated by any $k$-difference $\Delta_{g_{1}, \ldots, g_{k}}$ of a fixed order $k=k(l)$.

Here

$$
\Delta_{g_{1}, \ldots, g_{k}}:=\Delta_{g_{1}} \circ \ldots \circ \Delta_{g_{k}} \quad\left(g_{1}, \ldots, g_{k} \in G\right)
$$

and $\Delta_{g}(f)(x):=f(g x)-f(x)$.

In this paper we consider the case of a regular covering $M_{G}$ over a compact complex manifold $M$ with a free abelian transformation group $G$. Our approach is based on Algebraic Geometry technique and different from the methods of the above mentioned papers.

To present our results introduce

Definition 1.2. A regular covering $p: M_{G} \longrightarrow M$ over a compact complex manifold $M$ with a free abelian transformation group $G$ is said to be of the class $\mathcal{P} \mathcal{F}$ if all $\mathcal{P}_{l}\left(M_{G}\right)$ are finite dimensional.

The following result describes the structure of the space $\mathcal{P}_{l}\left(M_{G}\right)$ in the case $M_{G} \in \mathcal{P F}$. To its formulation we need a few notions.

Let $\Omega_{d}^{1}(M)$ be the space of $d$-closed holomorphic 1-forms on $M$. Define the subspace $\Omega_{d}^{1}(M ; G) \subset \Omega_{d}^{1}(M)$ as the set of all 1-forms $\omega$ such that $p^{*} \omega$ determines the trivial element of the cohomology class $H^{1}\left(M_{G}, \mathbf{C}\right)$. Let $\left\{\omega_{i}\right\}_{i=1}^{s}$ be a basis in this subspace. Consider the family

$$
\left\{f_{i}(z):=\int_{z_{0}}^{z} p^{*} \omega_{i}\right\}_{i=1}^{s}
$$

of holomorphic functions on $M_{G}$ and denote by $\mathcal{P}_{l}\left(f_{1}, \ldots, f_{s}\right)$ the subspace of the space $\mathbf{C}\left[f_{1}, \ldots, f_{s}\right]$ consists of polynomials of degree at most $l$.

Theorem 1.1. Let $M_{G} \in \mathcal{P} \mathcal{F}$. Then the space $\mathcal{P}_{l}\left(M_{G}\right)$ coincides with $\mathcal{P}_{l}\left(f_{1}, \ldots, f_{s}\right)$. Moreover, $\mathcal{P}_{l}\left(M_{G}\right)$ is annihilated by any $(l+1)$-difference.

To state the result in a sense converse to the previous one assumes that 
$M$ admits a holomorphic mapping $A$ in a complex torus CT $^{r}$ such that the induced homomorphism $A_{*}: H_{1}(M, \mathbf{Z}) \longrightarrow$ $H_{1}\left(\mathbf{C T}{ }^{r}, \mathbf{Z}\right)$ is surjective.

Consider now the regular covering $M_{G}$ over $M$ with the transformation group $G=H_{1}(M, \mathbf{Z}) / \operatorname{Ker} A_{*}\left(\cong \mathbf{Z}^{2 r}\right)$. According to the covering homotopy theorem there exists the covering to $A$ holomorphic mapping $A^{\prime}: M_{G} \longrightarrow$ $\mathbf{C}^{r}$.

Theorem 1.2. Under the previous assumption $M_{G} \in \mathcal{P F}$ and for every $f \in \mathcal{P}_{l}\left(M_{G}\right)$ there exists a holomorphic polynomial $p$ on $\mathbf{C}^{r}$ of degree at most $l$ such that $f=\left(A^{\prime}\right)^{*}(p)$.

Corollary 1.3. Let $M$ be a compact Kähler manifold and $M_{G}$ be a regular covering over $M$ with a free abelian group $G$. Then $M_{G} \in \mathcal{P F}$ and $\mathcal{P}_{l}\left(M_{G}\right)$ is linearly isomorphic to a subspace of the space $\mathcal{P}_{l}\left(\mathbf{C}^{N}\right)$ of holomorphic polynomials on $\mathbf{C}^{N}$ of degree at most $l$, where $N=\frac{1}{2} \operatorname{dim}_{\mathbf{C}} H_{1}(M, \mathbf{C})$.

To formulate the second corollary denote by $\tau(M)$ the transcendency degree of the field of meromorphic functions on $M$.

Corollary 1.4. Let $M$ be a compact complex manifold with $\tau(M) \geq$ $\operatorname{dim}_{\mathbf{C}} M-1$ and $G$ be a free abelian group. Then $M_{G} \in \mathcal{P} \mathcal{F}$.

Corollary 1.5. Let $M$ be a compact complex manifold of dimension 1 and genus $g$ and $M_{G}$ be a regular covering over $M$ with a free abelian group $G$. Then the space $\mathcal{H}_{l}\left(M_{G}\right)$ is finite dimensional and linearly isomorphic to the subsipace of harmonic polynomials on $\mathbf{C}^{g}$ of degree at most $l$ that are sums of holomorphic and antiholomorphic ones.

'The author is indebted to Professor V.Lin who raised the question discussed in this paper.

\section{Proof of Theorem 1.3 .}

Let $\tau$ be the canonical action of $G$ on the space $\mathcal{O}\left(M_{G}\right)$ of holomorphic functions on $M_{G}$ induced by the action of $G$ on $M_{G}$. By definition $\mathcal{P}_{l}\left(M_{G}\right)$ is irivariant with respect to $\tau$.

Lernma 2.1. $\tau$ is a unipotent representation, i.e., all eigenvalues of $\tau(g)$ equal 1 for every $g \in G$. 
Proof. Assume, to the contrary, that there exist a nonzero $g \in G$ and $f \in$ $\mathcal{P}_{l}\left(M_{G}\right)$, and $\lambda \neq 1$ such that

$$
\tau(g) f=\lambda f
$$

Firstly prove that $|\lambda|=1$. Otherwise we can assume without loss of generality that $|\lambda|>1$. In the case of a free abelian group the word metric $\rho$ is clearly satisfies $\rho\left(g^{n}\right)=|n| \rho(g)$. Therefore

$$
\left|\lambda^{n} f(z)\right|=\left|\left(\tau\left(g^{n}\right) f\right)(z)\right| \leq C(n \rho(g))^{l}
$$

and, hence,

$$
|f(z)| \leq \lim _{n \rightarrow \infty} \frac{C(n \rho(g))^{l}}{|\lambda|^{n}}=0 \quad(z \in V) .
$$

Since the fundamental compact $V$ has a nonempty interior, $f=0$ on $M_{G}$. This contradiction implies that $|\lambda|=1$. Now we prove that there exists a nonzero function $w \in \mathcal{P}_{l}\left(M_{G}\right)$ such that

(i) $\tau(h)(w)=\lambda(h) w$ for every $h \in G$;

(ii) $\tau(g)(w)=\lambda w$.

Really, let $W$ be the minimal subspace of $\mathcal{P}_{l}\left(M_{G}\right)$ containing the $f$ and invariant with respect to the action $\tau$. Since $W$ is the linear span of $\tau(G) f$, every $w \in W$ is an eigenvector for $\tau(g)$ with eigenvalue $\lambda$. As every finite dimensional representation of a free abelian group of finite rank, $\left.\tau\right|_{W}$ has logarithm (see, e.g., [G], ch. 8). Therefore it can be extended to a representation of the abelian Lie group $G \otimes \mathbf{R}$ into $G L(W)$. Indeed, let $\left\{e_{i}\right\}_{i=1}^{k}$ be a basis of $G$ and $\left\{f_{i}:=\log \left(\tau\left(e_{i}\right)\right)\right\}_{i=1}^{k}$. Then the required extension is given by

$$
\sum_{i=1}^{k} a_{i} f_{i} \mapsto \exp \left(\sum_{i=1}^{k} a_{i} f_{i}\right) \quad\left(a_{i} \in \mathbf{R}\right) .
$$

By the Lie theorem there exists a common eigenvector $w$ for the extended representation. It is clear that $w$ satisfies conditions $(i)$ and $(i i)$.

Repeating the arguments of the first part of the proof we conclude that $|\lambda(h)|=1$ for every $h \in G$. This implies that the plurisubharmonic function $|w(z)|$ is invariant with respect to the action of $G$ on $M_{G}$. Since $M$ is a compact complex manifold, the function has to be a constant. From here it follows that $w$ is a constant too and therefore $\lambda=1$. 
Lernma 2.2. Let $\operatorname{dim}_{C} \mathcal{P}_{l}\left(M_{G}\right)=n$. Then for every $f \in \mathcal{P}_{l}\left(M_{G}\right)$ and $g_{1}, \ldots, g_{n} \in G$

$$
\Delta_{g_{1}, \ldots, g_{n}}(f)=0 .
$$

Proof. Similarly to the construction of the previous proof we can define the extension of $\tau$ to a representation $\tau^{\prime}: G \otimes \mathbf{R} \longrightarrow G L_{n}(\mathbf{C})$. By Lemma $2.1 \tau^{\prime}$ is unipotent. In virtue of the Lie theorem (see, e.g., [OV]), $\tau^{\prime}$ is equivalent to a, representation $\tau^{\prime \prime}$ into the nilpotent matrix subgroup $N \subset G L_{n}(\mathbf{C})$ of upper triangular matrices with units on the diagonal. From here it follows that in an appropriate basis of $\mathcal{P}_{l}\left(M_{G}\right)$

$$
\Delta_{g_{1}, \ldots, g_{n}}=\prod_{i=1}^{n}\left(\tau^{\prime \prime}\left(g_{i}\right)-I\right)
$$

Now observe $\tau^{\prime \prime}\left(g_{i}\right)-I$ is an upper triangular matrix with zero diagonal. So the product of $n$ such matrices equals 0 .

Let $e_{1}, \ldots, e_{k}$ be a basis for $G \cong \mathbf{Z}^{k}$. Denote by $S_{l} \subset G$ the $l$-simplex along the group $G$, that is,

$$
S_{l}:=\left\{g=\sum_{i=1}^{k} s_{i} e_{i} ; s_{i} \geq 0, \sum_{i=1}^{k} s_{i} \leq l\right\},
$$

Lenma 2.3. Let $x \in M_{G}$ be a fixed point. Assume that a function $f \in$ $\mathcal{P}_{l}\left(M_{G}\right)$ equals 0 on the set $S_{n-1} x \subset M_{G}$. Then $f=0$ identically.

Proof. Let to the contrary $f \neq 0$. Then Lemma 2.2 implies the existence of a difference $\Delta_{g_{1}, \ldots, g_{l}}$ of the maximal order $l$ such that

(i) $f_{l}:=\Delta_{g_{1}, \ldots, g_{l}}(f) \neq 0$;

(ii) $\Delta_{g}\left(f_{l}\right)=0$ for every $g \in G$.

Easy computation shows that

$$
\Delta_{g_{1}, \ldots, g_{l}}=\sum_{i \in I} \pm \tau\left(h_{i}\right) \circ \Delta_{g_{1}^{i}, \ldots, g_{l}^{i}},
$$

where every $g_{s}^{i}$ coincides with one of $e_{1}, \ldots, e_{k}$. Therefore without loss of generality we can assume that in condition $(i)$ every element $g_{i}$ belongs to 
the basis of $G$. Because of the maximality of $l$ the condition $(i i)$ is also fulfilled for this choice of $g_{i}$. But $f_{l}(x)$ is a linear combination of $\tau\left(s_{j}\right) f(x)$ with $s_{j} \in S_{n-1}$. So $f_{l}(x)=0$ by the assumption of lemma. Moreover, in virtue of $(i i)$ the holomorphic function $f_{l}$ is invariant with respect to the action of $G$ and, therefore, is a constant. Hence, $f_{l}=0$ on $M_{G}$ and we obtain the contradiction with the maximality of $l$.

Lemma 2.4. $\mathcal{P}_{l}\left(M_{G}\right)$ is annihilated by any $(l+1)$-difference.

Proof. Let $x$ be a fixed point of $M_{G}$. Henceforth we identify the orbit $\{G x\}$ with the lattice $\mathbf{Z}^{k}$ of integer points in $\mathbf{R}^{k}$. Let $r_{x}: \mathcal{P}_{l}\left(M_{G}\right) \longrightarrow l_{\infty}\left(S_{n-1}\right)$ be the restriction to $S_{n-1}(x)$. According to Lemma $2.3 r_{x}$ is injective. We define now the linear mapping $i$ from $l_{\infty}\left(S_{n-1}\right)$ into the space $\mathcal{P}_{n-1}\left(\mathbf{R}^{k}\right)$ of complex-valued polynomials of degree at most $n-1$ as follows. Since $S_{n-1} \subset G$ is the set of uniqueness for $\mathcal{P}_{n-1}\left(\mathbf{R}^{k}\right)$ and $\operatorname{dim}_{\mathbf{C}} l_{\infty}\left(S_{n-1}\right)=$ $\operatorname{dim}_{\mathbf{C}} \mathcal{P}_{n-1}\left(\mathbf{R}^{k}\right)$, the operator $\left.p \mapsto p\right|_{S_{n-1}}$, where $p \in \mathcal{P}_{n-1}\left(\mathbf{R}^{k}\right)$, is invertible. Its inverse is the required operator $i$.

Let us choose now an arbitrary $f \in \mathcal{P}_{l}\left(M_{G}\right)$ and denote by $f_{x}$ the restriction of $f$ to $\{G x\}=\mathbf{Z}^{k}$. We show that

$$
\left.\left(i \circ r_{x}\right)\left(f_{x}\right)\right|_{\mathbf{Z}^{k}}=f_{x}
$$

Let $\phi:=\left.\left(i \circ r_{x}\right)\left(f_{x}\right)\right|_{\mathbf{Z}^{k}}-f_{x}$. Since $\left(i \circ r_{x}\right)\left(f_{x}\right) \in \mathcal{P}_{n-1}\left(\mathbf{R}^{k}\right)$, this polynomial is annihilated by any $n$-difference. According to Lemma $2.2 f_{x}$ is also annihilated by any $n$-difference and therefore the same is valid for $\phi$. Using the arguments from the proof of Lemma 2.3 we can state that $\phi$ is uniquely determined by its values on $S_{n-1}$. Since $\left.\phi\right|_{S_{n-1}}=0$ by the definition of $i$, this function equals 0 identically.

Let us consider now the polynomial $p_{f, x}:=\left(i \circ r_{x}\right)\left(f_{x}\right)$ and prove that its degree less than or equal to $l$. From here will follow that this polynomial is annihilated by any $(l+1)$-difference and therefore $f_{x}$ will be also annihilated by such differences. Because of the arbitrariness of $x$ this will prove the lemma.

Assume, to the contrary, that $\operatorname{deg}\left(p_{f, x}\right)>l$. By the definition of $\mathcal{P}_{l}\left(M_{G}\right)$ the function $\left.p_{f, x}\right|_{\mathbf{Z}^{k}}=f_{x}$ has $l$-polynomial growth at infinity. Let $s_{f, x}$ be the homogeneous part of $p_{f, x}$ of maximal degree. Then there exists a line $L:=\left\{\left(a_{1} t, \ldots, a_{k} t\right) \in \mathbf{R}^{k} ; t \in \mathbf{R}, a_{i} \in \mathbf{Z}, i=1, \ldots k\right\}$ such that $\left.s_{f, x}\right|_{L} \neq 0$. Otherwise $s_{f, x}=0$ on $\mathbf{Z}^{k}$ and hence it equals 0 on $\mathbf{R}^{k}$. According to our assumption $\left(\left.s_{f, x}\right|_{L}\right)(t)=c t^{d}$ with $d>l$ whereas this polynomial goes to 
infinity as $|t|^{l}$ in integer points of $L$. Therefore $c=0$ and this contradiction proves that $\operatorname{deg}\left(p_{f, x}\right) \leq l$.

We now are in a position to finish the proof of Theorem 1.1 by induction on $l$. Namely, we introduce the set $\mathcal{D}_{l}\left(M_{G}\right)$ of holomorphic on $M_{G}$ functions annihilated by any $(l+1)$-difference and prove by induction that any element of this set can be represented as a polynomial in $f_{1}, \ldots, f_{s}$ of degree at most $l$. Since every such polynomial belongs to $\mathcal{P}_{l}\left(M_{G}\right)$, this result together with Lemma 2.4 will complete the proof of the theorem.

The basis of the induction is

Lemma 2.5. Let $f \in \mathcal{D}_{1}\left(M_{G}\right)$. Then $f$ is a linear polynomial in $f_{1}, \ldots, f_{s}$.

Proof. Since $f$ is annihilated by any 2-difference, $f(g x)-f(x)$ is a constant for a fixed $g \in G$. Therefore $d f$ is a $G$-invariant $d$-closed holomorphic 1-form on $M_{G}$. Hence there exists a form $\eta \in \Omega_{d}^{1}(M ; G)$ such that its pullback to $M_{G}$ coincides with $d f$. From here it follows that $f$ up to an additive constant can be represented as a linear combination of the functions $f_{i}$, i.e., $f \in \mathcal{P}_{1}\left(f_{1}, \ldots, f_{s}\right)$.

A.ssume now that the statement of the induction has already been proved for $\mathcal{D}_{l-1}\left(M_{G}\right)$ and prove it for $\mathcal{D}_{l}\left(M_{G}\right)$.

Let $F$ be the maximal free abelian subgroup of the homology group $H_{1}(M, \mathbf{Z})$ and $R \subset F$ determined by $F / R \cong G$. Since $\int_{\gamma} \omega=0$ for every $\omega \in \Omega_{d}^{1}(M ; G)$ and $\gamma \in R$, we get

$$
\operatorname{dim}_{\mathbf{C}} \Omega_{d}^{1}(M ; G) \leq \frac{1}{2} \operatorname{rank}(G) .
$$

In addition, $\Omega_{d}^{1}(M ; G)$ can be identified with a subspace of the space dual to $F \otimes \mathbf{C} \cong H_{1}(M, \mathbf{C})$. Therefore there exists a subgroup $A \subset F$ of

$$
\operatorname{rank}(A)=2 \operatorname{dim}_{\mathbf{C}} \Omega_{d}^{1}(M ; G)
$$

such that the set $\Gamma$ of the vectors $\left(\int_{\alpha} \omega_{1}, \ldots, \int_{\alpha} \omega_{s}\right) \in \mathbf{C}^{s} \quad(\alpha \in A)$ satisfies the condition

$\Gamma$ is a lattice of rank $2 s$ in $\mathbf{C}^{s}$. 
Since $A \bigcap R=\{0\}$, the natural surjection $F \longrightarrow F / R \cong G$ imbeds $A$ into $G$. So we can consider $A$ as a subgroup of $G$. Denote by $E$ the quotient group $G / A$ and consider the regular covering $p_{E}: M_{E} \longrightarrow M$ with the transformation group $E$. Because of the choice of $E$ there exists a regular covering $p_{1}: M_{G} \longrightarrow M_{E}$ with the transformation group $A$. Condition (2.2) implies that the multivalued function

$$
t(z):=\left(\int_{z_{0}}^{z} p_{E}^{*} \omega_{1}, \ldots, \int_{z_{0}}^{z} p_{E}^{*} \omega_{s}\right) \quad\left(z \in M_{E}\right)
$$

where $z_{0} \in M_{E}$ is fixed, determines the holomorphic mapping into the complex torus $\mathbf{C}^{s} / \Gamma$. Then one naturally defines the mapping $t^{\prime}: M_{G} \longrightarrow \mathbf{C}^{s}$, which covers $t$ and is clear to be equivariant with respect to the actions of $A$ on $M_{G}$ and $\Gamma$ on $\mathbf{C}^{s}$. Therefore there exists the set $\left\{g_{1}, \ldots, g_{s}\right\}$ of $\mathbf{C}$-linear independent holomorphic polynomials of degree 1 on $\mathbf{C}^{s}$ such that

$$
\left(t^{\prime}\right)^{*}\left(g_{i}\right)=f_{i}:=\int_{y_{0}}^{y} p^{*} \omega_{i} \quad(1 \leq i \leq s) .
$$

Here $y, y_{0} \in M_{G}$ and $y_{0}$ is fixed. Using an affine transformation we can regard $g_{i}$ as the coordinate functions of $\mathbf{C}^{s}$. Assume that the similar relation is valid for every $f$ annihilated by any $l$-difference, that is, there exists a holomorphic polynomial $p \in \mathcal{P}_{l-1}\left(\mathbf{C}^{s}\right)$ of degree at most $l-1$ such that

$$
\left(t^{\prime}\right)^{*}(p)=f
$$

In view of the previous relation this means that $f$ is a polynomial in $f_{i}$ $(1 \leq i \leq s)$ of degree at most $l-1$.

To finish the proof it remains therefore to establish the corresponding statement for $f \in \mathcal{D}_{l}\left(M_{G}\right)$. To this end choose an arbitrary $x \in M_{G}$ and consider the orbit $\{A x\} \subset M_{G}$, which we can identify with the lattice $\mathbf{Z}^{2 s} \subset$ $\mathbf{R}^{2 s}$. Consider now the $k$-simplex $S_{k}$ along the group $A \cong \mathbf{Z}^{2 s}$, see (2.1) for its definition. In this definition $e_{1}, \ldots, e_{2 s}$ is the standard basis for $\mathbf{Z}^{2 s}$. As a consequence of the statement of induction for $(l-1)$ we get $S_{l-1}$ is a set of uniqueness for $\mathcal{D}_{l-1}\left(M_{G}\right)$. Then $S_{l}$ is a set of uniqueness for $\mathcal{D}_{l}\left(M_{G}\right)$. For if $h \in \mathcal{D}_{l}\left(M_{G}\right)$ equals 0 on $S_{l}$ then clearly the function $h_{i}(z):=h\left(e_{i} z\right)-h(z)$ is an element of $\mathcal{D}_{l-1}\left(M_{G}\right)$ equals 0 on $S_{l-1}$. Therefore $h_{i}=0$ for every $i$ and thus $h$ is $A$-invariant. The same is clear to be correct for $h^{g}(z):=h(g z)-h(z)$ $(g \in G)$. Moreover, $h^{g} \in \mathcal{D}_{l-1}\left(M_{G}\right)$ and therefore by induction there exists a polynomial $p^{g} \in \mathcal{P}_{l-1}\left(\mathbf{C}^{s}\right)$ such that

$$
\left(t^{\prime}\right)^{*}\left(p^{g}\right)=h^{g} .
$$


Then for every $a \in A$ we get

$$
\left(t^{\prime}\right)^{*}\left(p^{g}(a+z)-p^{g}(z)\right)=h^{g}(a z)-h^{g}(z)=0,
$$

that is, $p^{g}$ is $A$-invariant. Thus $p^{g}$ is a constant and so $h^{g}$ is. This means that $h \in \mathcal{D}_{1}\left(M_{G}\right)$ and by Lemma $2.5 h=0$. By this one has proved that $S_{l}$ is a set of uniqueness for $\mathcal{D}_{l}\left(M_{G}\right)$.

Using this fact we can find for the function $f \in \mathcal{D}_{l}\left(M_{G}\right)$ a polynomial $p_{f}$ on $\mathbf{R}^{2 s}$ of degree at most $l$ such that

$$
p_{f}=f \quad \text { on } \mathbf{Z}^{2 s},
$$

see Lemma 2.4. Since $t^{\prime}$ is equivariant with respect to the actions of $A$ on $M_{G}$ and $\Gamma$ on $\mathbf{C}^{s}$, we can identify $\mathbf{R}^{2 s}$ with $\mathbf{C}^{s}$ and $\mathbf{Z}^{2 s}$ with $\Gamma$. These identifications and the assumption of the induction applying to $f$ get that any clifference $\Delta_{a}\left(p_{f}\right):=p_{f}(a+z)-p_{f}(z)$ is a holomorphic on $\mathbf{C}^{s}$ polynomial of degree at most $(l-1)$. Then $(0,1)$-form $\bar{\partial} p_{f}$ is $\Gamma$-invariant and therefore by the Hodge decomposition

$$
\bar{\partial} p_{f}=\sum_{i=1}^{s} a_{i} \overline{d z_{i}}+\bar{\partial} w
$$

where $w$ is $\Gamma$-invariant and $z_{1}, \ldots, z_{s}$ stand for the standard coordinate functions on $\mathbf{C}^{s}$. This leads to the identity

$$
p_{f}(z)=\sum_{i=1}^{s} a_{i} \overline{z_{i}}+w(z)+u(z),
$$

where $u$ is a holomorphic on $\mathbf{C}^{s}$ function of $l$-polynomial growth, that is, $u$ is a holomorphic polynomial of degree at most $l$. From here and $\Gamma$-invariance it follow's that $w$ has to be a constant. Consider now the function $f-\left(t^{\prime}\right)^{*}(u)$. Because of the above identity and the interpolating property of $p_{f}$ any 2difference of the function equals 0 on the orbit $\{A x\}$. Bearing in mind that such a difference belongs to $\mathcal{D}_{l-2}\left(M_{G}\right)$ and applying the statement of the induction we obtain that any 2-difference of $f-\left(t^{\prime}\right)^{*}(u)$ equals 0 on $M_{G}$. Therefore $f-\left(t^{\prime}\right)^{*}(u) \in \mathcal{D}_{1}\left(M_{G}\right)$ and by Lemma 2.5 there exists a linear holomorphic on $\mathbf{C}^{s}$ polynomial $q$ such that

$$
f=\left(t^{\prime}\right)^{*}(u+q) .
$$

This proves the statement of the induction and completes the proof of Theorem 1.1. 


\section{Properties of PF Spaces.}

Here we collect a few results which will be used in the sequel. Some of them are interesting in their own right. To formulate the first result consider a compact complex manifold $M$ possessing the following property:

There exist collections $\left\{V_{s}\right\}_{s \in S}$ of complex compact manifolds and holomorphic mappings $\left\{\phi_{s}: V_{s} \longrightarrow M\right\}_{s \in S}$ such that

(i) $\phi_{s}: V_{s} \longrightarrow M$ induces the surjective homomorphism $\left(\phi_{s}\right)_{*}$ : $H_{1}\left(V_{s}, \mathbf{Z}\right) \longrightarrow H_{1}(M, \mathbf{Z})$

(ii) the union of all $\phi_{s}\left(V_{s}\right)$ has nonempty interior.

Denote by $F_{s}$ and $F$ the maximal free abelian subgroups of $H_{1}\left(V_{s}, \mathbf{Z}\right)$ and $H_{1}(M, \mathbf{Z})$, respectively.

Proposition 3.1. Assume that for every $s$ the regular covering $W_{s}$ over $V_{s}$ with the transformation group $F_{s}$ belongs to $\mathcal{P} \mathcal{F}$. Then the regular covering $M_{F}$ over $M$ with the transformation group $F$ belongs to $\mathcal{P} \mathcal{F}$.

Proof. By assumption (i) the image $\left(\phi_{s}\right)_{*}\left(F_{s}\right)$ can be represented as $F \oplus G_{s}$ with a finite subgroup $G_{s}$ contained in the torsion subgroup of $H_{1}(M, \mathbf{Z})$. Let $H_{s}:=\left(\phi_{s}\right)_{*}^{-1}(F)$ and consider a subgroup $E_{s}$ in $F_{s}$ such that

$$
H_{s} \bigcap E_{s}=\{0\}, \quad H_{s}+E_{s} \text { has finite index in } F_{s} .
$$

Let $M_{1}$ be the regular covering over $M$ with the transformation group $H_{1}(M, \mathbf{Z})$. By assumption $(i)$ and the covering homotopy theorem there is a holomorphic mapping $\phi_{s}^{\prime}: W_{s} \longrightarrow M_{1}$ which covers $\phi_{s}$ and is equivariant with respect to the actions of $F_{s}$ on $W_{s}$ and $\left(\phi_{s}\right)_{*}\left(F_{s}\right)$ on $M_{1}$. Similarly for the covering $M_{F}$ there exists the covering mapping $r: M_{1} \longrightarrow M_{F}$ equivariant with respect to the action $F$ on $M_{1}$ and $M_{F}$.

Let now $r^{*} f$ be the pullback to $M_{1}$ of a function $f \in \mathcal{P}_{l}\left(M_{F}\right)$ and $f^{\prime}:=\left(\phi_{s}^{\prime}\right)^{*}\left(r^{*} f\right)$. In virtue of the definition of $E_{s}$ and the equivariance of $\phi_{s}^{\prime}$ and $r$ the function $f^{\prime}$ is invariant with respect to the action of the group $E_{s}$. Since the free abelian group $F$ is quotient group of $H_{s}$ and $f \in \mathcal{P}_{l}\left(M_{F}\right)$, the function $f^{\prime}$ has $l$-polynomial growth with respect to the action of $H_{s}$. In addition, $H_{s} \oplus E_{s}$ has a finite index in $F_{s}$ and therefore $f^{\prime}$ has also $l$ polynomial growth with respect to the action of $F_{s}$. By the assumptions of the proposition $W_{s} \in \mathcal{P F}$. So applying the statement of Theorem 1.1 to $f^{\prime}$ 
we obtain that any $(l+1)$-difference $\Delta_{h_{1}, \ldots, h_{l+1}}$ with $h_{i} \in H_{s}$ annihilates $f^{\prime}$. Since the image of $\left(\phi_{s}\right)_{*}\left(H_{s}\right)$ coincides with $F$, the equivariance arguments imply the restriction of $f$ to $\phi_{s}^{\prime}\left(W_{s}\right)$ is annihilated by any $(l+1)$-difference. However, from assumption $(i i)$ it follows that the union of all $\phi_{s}^{\prime}\left(W_{s}\right)$ has nonempty interior. So holomorphicity of $f$ implies that any $(l+1)$-difference annihilates $f$, i.e., $f \in \mathcal{D}_{l}\left(M_{F}\right)$. It was proved in Theorem 1.1 that the latter vector space admits an imbedding into the space of holomorphic polynomials of degree at most $l$ defined on some $\mathbf{C}^{s}$. This means that $M_{F} \in \mathcal{P F}$.

Let $h: X \longrightarrow Y$ be a holomorphic surjective mapping of compact complex manifolds. Assume that fiber $h^{-1}(y)$ over the generic point $y \in Y$ is finite. Let $k:=\operatorname{deg}(h)$ be degree of $h$, that is, the number of points in $h^{-1}(y)$ over the generic $y \in Y$. Denote by $H$ and $G$ the maximal free abelian subgroups of $H_{1}(Y, \mathbf{Z})$ and $H_{1}(X, \mathbf{Z})$, respectively. Assume also that

(i) $h_{*}: H_{1}(X, \mathbf{Z}) \longrightarrow H_{1}(Y, \mathbf{Z})$ maps $G$ into $H$;

(ii) there is a surjective homomorphism $\tau$ from $H$ into a free abelian group $F$.

Put $G^{\prime}:=\left(\tau \circ h_{*}\right)(G)$.

Finally denote by $X_{G^{\prime}}$ and $Y_{F}$ regular coverings over $X$ and $Y$ with the transformation groups $G^{\prime}$ and $F$, respectively.

Proposition 3.2. Let $\mathcal{P}_{k l}\left(Y_{F}\right)$ be finite dimensional. Then $\mathcal{P}_{l}\left(X_{G^{\prime}}\right)$ has finite dimension, as well.

Proof. Surjectivity of $h$ implies $h_{*}\left(\pi_{1}(X)\right)$ is of finite index in $\pi_{1}(Y)$. In particular, $G^{\prime}$ is of finite index in $F$. From here and the covering homotopy theorem follow that there exists the covering to $h$ holomorphic mapping $h^{\prime}: X_{G^{\prime}} \longrightarrow Y_{F}$ such that $h^{\prime}$ is a proper surjective mapping of the degree $k$ equivariant with respect to the actions of $G^{\prime}$ on $X_{G^{\prime}}$ and $Y_{F}$. Consider the Stein factorization of $h^{\prime}$, that is, the analytical variety $V$ and holomorphic mappings $h_{1}: X_{G^{\prime}} \longrightarrow V$ and $h_{2}: V \longrightarrow Y_{F}$ such that $h_{2}$ is a finite branched covering over $Y_{F}$ of degree $k$ and $h_{1}$ has compact connected fibers, and $h^{\prime}=h_{2} \circ h_{1}$. Let $f$ be a holomorphic function on $X_{G^{\prime}}$. Then $f$ is clear to be constant on any fiber of $h_{1}$ and therefore there exists a holomorphic on $V$ function $f^{\prime}$ such that $h_{1}^{*}\left(f^{\prime}\right)=f$.

We are now in a position to prove the proposition. To this end we construct a smooth immersion of $\mathcal{P}_{l}\left(X_{G^{\prime}}\right)$ into a finite dimensional space. 
Since such immersion preserves dimension we obtain from here the required result. To construct the immersion fix a function $f \in \mathcal{P}_{l}\left(X_{G^{\prime}}\right)$. Then there exists a holomorphic on $V$ function $f^{\prime}$ such that $h_{1}^{*}\left(f^{\prime}\right)=f$. Let $x \in Y_{F}$ be the generic point and $h_{2}^{-1}(x)=\left\{x_{1}, \ldots, x_{k}\right\}$. Consider the polynomial

$$
\prod_{i=1}^{k}\left(t-f^{\prime}\left(x_{i}\right)\right)=\sum_{i=1}^{k} s_{i}\left(f^{\prime}\right)(x) t^{i} .
$$

The symmetric polynomials $s_{i}\left(f^{\prime}\right)$ are correctly defined outside of a divisor $D \subset Y_{F}$ and locally bounded in a neighborhood of every point of $D$. So by the Riemann theorem they can be extended to $Y_{F}$ as holomorphic functions for which we preserve the same notations. Moreover, in virtue of equivariance of $h^{\prime}$ with respect to the cocompact actions of $G^{\prime}$ on $X_{G^{\prime}}$ and $Y_{F}$, the function $s_{i}\left(f^{\prime}\right)$ belongs to $\mathcal{P}_{i l}\left(Y_{F}\right)$. Let now

$$
i(f):=\left(s_{1}\left(f^{\prime}\right), \ldots, s_{k}\left(f^{\prime}\right)\right) \quad\left(f \in \mathcal{P}_{l}\left(X_{G^{\prime}}\right)\right) .
$$

Since the Gâteaux derivative of $f \mapsto s_{1}\left(f^{\prime}\right)$ is readily seen to be different from $0, i$ has no critical points, i.e., the smooth mapping $i$ is an immersion into the direct product $\mathcal{P}_{l}\left(Y_{F}\right) \times \ldots \times \mathcal{P}_{k l}\left(Y_{F}\right)$. By the assumption of the proposition the latter is finite dimensional. This completes the proof.

Remark 3.3. Literally repeating these arguments we obtain the same result in the case where $h: X \longrightarrow Y$ is a finite branched covering over a compact complex manifold $Y$ with irreducible $X$.

Let $h: X \longrightarrow Y$ be a holomorphic surjective mapping of compact complex manifolds with connected fibers. Denote a fiber $h^{-1}(y)$ over the generic point $y \in Y$ by $Z_{y}$ and the maximal free abelian subgroup of $H_{1}\left(Z_{y}, \mathbf{Z}\right)$ by $H_{y}$. Let $G$ and $F$ be the maximal free abelian subgroups of $H_{1}(Y, \mathbf{Z})$ and $H_{1}(X, \mathbf{Z})$, respectively. Finally denote by $Y_{G}, X_{F}$ and $Z_{y}^{\prime}$ the regular coverings over $X, Y$ and $Z_{y}$ with the transformation groups $G, F$ and $H_{y}$, respectively.

Proposition 3.3. Let $Y_{G} \in \mathcal{P F}$ and $Z_{y}^{\prime} \in \mathcal{P F}$ for the generic $y$. Then $X_{F} \in \mathcal{P F}$.

Proof. Let $K$ be an arbitrary fiber of $h: X \longrightarrow Y$ and $i^{K}: K \longrightarrow X$ be the natural imbedding into $X$. 
Lemma 3.4. There exists an injective linear mapping

$$
s: H_{1}(Y, \mathbf{R}) \longrightarrow H_{1}(X, \mathbf{R})
$$

such that $H_{1}(X, \mathbf{R})$ is isomorphic to $s\left(H_{1}(Y, \mathbf{R})\right) \oplus\left(i^{K}\right)_{*}\left(H_{1}(K, \mathbf{R})\right)$.

Proof. By the Sard theorem there exists a proper closed analytical subset $D \subset Y$ such that $h: X \backslash h^{-1}(D) \longrightarrow Y \backslash D$ is a $C^{\infty}$ fiber bundle. To begin with let $K$ be a fiber of this bundle. Since $X$ is a connected complex manifold, the imbedding $X \backslash h^{-1}(D) \longrightarrow X$ induces a surjective homomorphism $\tau$ of the corresponding 1-homology groups. Moreover, the exactness of the homotopy sequence for the above constructed fiber bundle leads to existence of an injective linear mapping $s^{\prime}: H_{1}(Y \backslash D, \mathbf{R}) \longrightarrow H_{1}\left(X \backslash h^{-1}(D), \mathbf{R}\right)$ such that

(i)

$$
H_{1}\left(X \backslash h^{-1}(D), \mathbf{R}\right)=s^{\prime}\left(H_{1}(Y \backslash D, \mathbf{R})\right) \oplus\left(i^{K}\right)_{*}\left(H_{1}(K, \mathbf{R})\right) ;
$$

(ii) the mapping $\tau \circ s^{\prime}$ equals 0 on

$$
\operatorname{Ker}\left\{H_{1}(Y \backslash D, \mathbf{R}) \longrightarrow H_{1}(Y, \mathbf{R})\right\} .
$$

The mapping of the homology groups in $(i i)$ is induced by the natural imbedding. It follows from (ii) that $\tau \circ s^{\prime}$ determines the injective mapping $s: H_{1}(Y, \mathbf{R}) \longrightarrow H_{1}(X, \mathbf{R})$. In addition, $(i)$ implies that $s\left(H_{1}(Y, \mathbf{R})\right) \bigcap\left(i^{K}\right)_{*}\left(H_{1}(K, \mathbf{R})\right)=\{0\}$ and direct sum of these two vector spaces coincides with $H_{1}(X, \mathbf{R})$. It proves the result in this case. In particular, in this situation we obtained that

$$
\left(i^{K}\right)_{*}\left(H_{1}(K, \mathbf{R})\right)=\operatorname{Ker}(h)_{*} .
$$

Let now $K$ be an arbitrary fiber of $h$. Then the triangulation theorem for analytical sets implies that there exists an open neighborhood $U$ of $K$ such that $K$ is a deformation retract of $U$. By compactness arguments $U$ contains a fiber $E$ of the above constructed $C^{\infty}$ fiber bundle. Using this fact we will prove (3.1) for such $K$. Then the above defined imbedding $s$ determines the required decomposition of $H_{1}(X, \mathbf{R})$. To prove (3.1) assume to the contrary that $\left(i^{K}\right)_{*}\left(H_{1}(K, \mathbf{R})\right) \neq \operatorname{Ker}(h)_{*}$. Since $K$ is a deformation retract of $U$, from here it follows that there exist a $d$-closed 1-form $\omega$ on $X$ and an element $\gamma \in\left(i^{E}\right)_{*}\left(H_{1}(E, \mathbf{R})\right)$ such that $\left.\omega\right|_{U}$ is $d$-exact but $\int_{\gamma} \omega \neq 0$. However, $E \subset U$ and we get a contradiction. 
Denote now by $A(K)$ the maximal free abelian subgroup of $\left(i^{K}\right)_{*}\left(H_{1}(K, \mathbf{Z})\right)$. As a consequence of the lemma we obtain that $A(K) \oplus s(G)$ is of finite index in $F$.

Lemma 3.5. Let $H$ be the maximal free abelian subgroup of $\operatorname{Ker}(h)_{*}$. Then there exists a subgroup $L \subset H$ of a finite index such that for every fiber $K$ of $h$

$$
L \subset A(K) \subset H \text {. }
$$

Proof. Let $E$ be a fiber of the above constructed $C^{\infty}$ bundle over $Y \backslash D$. It is clear that $A(E)$ is independent of $E$ and has a finite index in $H$. Denote this group by $L$. For an arbitrary fiber $K$ choose as above an open neighborhood $U$ such that $K$ is a deformation retract of $U$ and $U$ contains $E$. Then $L=A(E)$ is to be contained in $A(K) \subset H$.

Consider now the regular covering $p: X_{E} \rightarrow X$ with the finite transformation group $E:=F /(L \oplus s(G))$. Let $\left(V, f_{1}, f_{2}\right)$ be the Stein factorization of the mapping $h \circ p: X_{E} \longrightarrow Y$. So $V$ is an analytic space, $f_{1}: X_{E} \longrightarrow V$ is a surjective holomorphic mapping with connected fibers and $f_{2}: V \longrightarrow Y$ is a finite branched covering. According to definition of $E$ the composite mapping

$$
H_{1}\left(X_{E}, \mathbf{Z}\right) \stackrel{\left(f_{1}\right)_{*}}{\longrightarrow} H_{1}(V, \mathbf{Z}) \stackrel{\left(f_{2}\right)_{*}}{\longrightarrow} H_{1}(Y, \mathbf{Z}) \longrightarrow G
$$

is surjective. Therefore there exists the regular covering $V_{G}$ over $V$ with the transformation group $G$. Then the covering to $f_{2}$ mapping $f_{2}^{\prime}: V_{G} \longrightarrow Y_{G}$ is finite branched and equivariant with respect to the actions of $G$ on $V_{G}$ and $Y_{G}$. Note that the generic fiber of $f_{1}$ is biholomorphic to the generic fiber of $h: X \longrightarrow Y$. The same is clear to be true for the generic fiber of the covering to $f_{1}$ mapping $f_{1}^{\prime}: X_{G}^{\prime} \longrightarrow V_{G}$, where $X_{G}^{\prime}$ is the covering over $X_{E}$ with the transformation group $G$. Finally, consider the covering $r: X_{F} \longrightarrow X_{G}^{\prime}$ with the transformation group $L$. According to Lemmae 3.4 and 3.5 we get:

$$
f_{1}^{\prime} \circ r: X_{F} \longrightarrow V_{G} \text { has connected fibers and } L \text { acts cocompactly }
$$
on every fiber of $f_{1}^{\prime} \circ r$.

Futher fix an $x \in Y_{G}$ such that its preimage $\left(f_{2}^{\prime} \circ f_{1}^{\prime} \circ r\right)^{-1}(x)$ in $X_{F}$ is a smooth submanifold. Consider the set $S_{l k} x \subset Y_{G}$, where $k:=\operatorname{deg}\left(f_{2}\right)$ and $S_{l k}$ is the $l k$-simplex along the group $G$ (see (2.1)). Denote by $S$ preimage of $S_{l k} x$ under the mapping $f_{2}^{\prime}$. So, $S \subset V_{G}$ and is finite. Let $R$ be a finite 
subset of $X_{F}$ such that $\left.f^{\prime}\right|_{R}$ is a bijection onto $S$. Finally, consider the set $S_{l} R$, where $S_{l}$ is the $l$-simplex along the group $L$. To complete the proof of the proposition it remains to establish the following result.

Lernma 3.6. Let $f \in \mathcal{P}_{l}\left(X_{F}\right)$ equal 0 on the set $S_{l} R$. Then $f$ equals 0 on $X_{F}$.

Proof. Assume, to the contrary, that $f \neq 0$. Let $Z \subset X_{F}$ be the generic fiber: of $f_{1}^{\prime} \circ r$. Then as above the group $L$ acts cocompactly on the complex manifold $Z$ and the quotient manifold $Z / L$ is biholomorphic to the generic fiber: of $h: X \longrightarrow Y$. By the assumption of the proposition the space $\mathcal{P}_{l}(Z)$ of holomorphic $l$-polynomial growth functions with respect to the action of $L$ is finite dimensional. In virtue of Theorem 1.1 every $(l+1)$-difference defined with the help of elements of $L$ annihilates $\mathcal{P}_{l}(Z)$. Since $\left.f\right|_{Z}$ belongs to $\mathcal{P}_{l}(Z)$ and union of generic fibers of $f_{1}^{\prime} \circ r$ has nonempty interior, $f$ is annihilated by each $(l+1)$-difference defined by elements of $L$. This and the arguments from the proof of Lemma 2.3 applying to the $f \neq 0$ lead to existence of a difference $\Delta_{g_{1}, \ldots, g_{s}}(s \leq l)$ with $g_{i}$ from the set of generators of $L$ such that

$$
Q:=\Delta_{g_{1}, \ldots, g_{s}} f \neq 0 \text { and } \Delta_{g} Q=0, \text { for every } g \in L .
$$

Therefore for each fiber $K$ of $f_{1}^{\prime}$ or the function $\left.Q\right|_{K}$ is invariant with respect to the action of $L$. But $K / L$ is a compact connected complex variety and so $\left.Q\right|_{K}$ is a constant. Consequently, there exists a holomorphic on $V_{G}$ function $Q^{\prime} \neq 0$ such that $\left(f_{1}^{\prime} \circ r\right)^{*}\left(Q^{\prime}\right)=Q$. Since $f_{1}^{\prime} \circ r$ is equivariant with respect to the action of $G$, the function $Q^{\prime}$ belongs to $\mathcal{P}_{l}\left(V_{G}\right)$. By the assumption of the lemma $Q=\Delta_{g_{1}, \ldots, g_{s}} f$ equals 0 on the set $R$ and therefore $Q^{\prime}=0$ on $S$. Show now that $Q^{\prime}=0$ identically that will lead to contradiction with $f \neq 0$. To establish this consider the symmetric polynomials $s_{i}\left(Q^{\prime}\right)$ defined by

$$
\prod_{i=1}^{k}\left(t-Q^{\prime}\left(y_{i}\right)\right)=\sum_{i=1}^{k} s_{i}\left(Q^{\prime}\right)(y) t^{i}
$$

where $\left\{y_{1}, \ldots, y_{k}\right\}$ is a fiber of $f_{2}^{\prime}: V_{G} \longrightarrow Y_{G}$ over the generic $y$. As in the proof of Proposition $3.2 s_{i}\left(Q^{\prime}\right)$ can be extended to a holomorphic on $Y_{G}$ function from $\mathcal{P}_{i l}\left(Y_{G}\right)$. Moreover, $s_{i}\left(Q^{\prime}\right)$ equals 0 on the set $S_{k l} x$. Since $Y_{G} \in$ $\mathcal{P F}$, the arguments from proof of Theorem 1.1 applying to functions $s_{i}\left(Q^{\prime}\right)$ yield these functions equal 0 identically $(i \leq k)$. This implies immediately $Q^{\prime}=0$. 
To finish the proof of the proposition consider the mapping from $\mathcal{P}_{l}\left(X_{F}\right)$ into $l^{\infty}\left(S_{l} R\right)$ defined by $\left.f \mapsto f\right|_{S_{l} R}$. The above lemma states that it is injective. Therefore $\operatorname{dim}_{\mathrm{C}} \mathcal{P}_{l}\left(M_{G}\right)<\infty$.

\section{Proof of Theorem 1.4 .}

The proof is divided into three parts. First we prove the theorem in the case of a compact complex curve. Then we consider the case of a compact projective manifold and, finally, finish the proof.

Let $M$ be a compact complex curve of genus $g$. The Albanese mapping $A$ imbeds $M$ into its Jacobi manifold biholomorphic to a complex torus CT $^{g}$ and induces the surjective homomorphism of the corresponding fundamental groups. As well-known the regular covering $M_{G}$ over $M$ with the transformation group $G=A_{*}\left(\pi_{1}(M)\right) \cong \mathrm{Z}^{2 g}$ is the maximal abelian covering over $M$. Therefore it suffices to prove the theorem for the case of $M_{G}$. Let $A^{\prime}: M_{G} \longrightarrow \mathbf{C}^{g}$ be the holomorphic imbedding that covers $A$. Since the Jacobi manifold of the curve $M$ is projective, there exists a very ample line bundle $L$ over $\mathbf{C T}^{g}$. According to the Kodaira imbedding theorem we may think of $\mathbf{C T}^{g}$ as imbedded in some projective space $\mathbf{C P}^{N}$ and of $L$ as the restriction to $\mathbf{C T}^{g}$ of the hyperplane bundle with the standard positively curved metric. Then zero loci of sections of $L$ are hyperplane sections of $\mathbf{C T}^{g}$. By Bertini's theorem, the generic linear subspace of codimension $g-1$ intersects $\mathbf{C T}^{g}$ transversely in a smooth curve $C$. By the Lefschetz hyperplane theorem, $C$ is connected and the map $\pi_{1}(C) \longrightarrow \pi_{1}\left(\mathbf{C T}^{g}\right)$ is surjective. Let $C_{G}$ be the regular covering with the above defined transformation group $G \cong \pi_{1}\left(\mathbf{C T}^{g}\right)$. Then there exists a holomorphic imbedding of $C_{G}$ into $\mathbf{C}^{g}$ and we will consider $C_{G}$ as a submanifold of $\mathbf{C}^{g}$.

Proposition 4.1. For every integer $l \geq 0$ there exists a very ample bundle $L$ over $\mathbf{C T}^{g}$ such that $\mathcal{P}_{(g !) l}\left(C_{G}\right)$ is finite-dimensional. Moreover, for every $f \in \mathcal{P}_{s}\left(C_{G}\right)$ with $s \leq(g !) l$ there exists a holomorphic polynomial $p$ on $\mathbf{C}^{g}$ of degree at most $s$ such that its restriction to $C_{G}$ coincides with $f$.

Proof. Let

$$
\rho\left(z_{1}, \ldots, z_{g}\right):=\left(1+\sum_{i=1}^{g}\left|z_{i}\right|^{2}\right)^{1 / 2}
$$


Obviously, $f \in \mathcal{P}_{l}\left(C_{G}\right)$ if and only if

$$
|f(z)| \leq C(f) \rho(z)^{l} \quad\left(z \in C_{G}\right) .
$$

Let $\omega:=\sum_{i=1}^{g} d z_{i} \Lambda d \overline{z_{i}}$ be the Kähler form on $\mathbf{C T}^{g}$ defining the Euclidean metric and $d V_{C}$ and $d V$ be the volume forms determined by $\omega$ on $C$ and $\mathbf{C T}^{g}$, respectively. We preserve the same notations for the pullback volume forms on $C_{G}$ and $\mathbf{C}^{g}$ and the pullback Kähler form on $\mathbf{C}^{g}$.

Lemma 4.2. For every $f \in \mathcal{P}_{s}\left(C_{G}\right)$ the inequality

$$
\int_{C_{G}}|f|^{2} \rho^{-t} d V_{C}<\infty
$$

holds with $t:=2 s+2 g+1$.

Proof. Since $d V_{C}$ is invariant with respect to the action of $G$, the integrability condition can be rewritten as

$$
\sum_{g \in G} \int_{V_{0}}|f(g z)|^{2} \rho(g z)^{-t} d V_{C}<\infty
$$

where $V_{0}$ is a compact fundamental domain. Because of compactness of $V_{0}$

$$
C \rho(g) \leq \rho(g z) \quad\left(g \in G, z \in V_{0}\right)
$$

for some constant $C>0$. Together with (4.1) this gives for $t>2 s$ :

$$
\sum_{g \in G} \int_{V_{0}}|f(g z)|^{2} \rho(g z)^{-t} d V_{C} \leq C^{\prime} \sum_{g \in G} \rho(g)^{2 s-t} \operatorname{vol}\left(V_{0}\right) .
$$

The righthand side is finite if $2 s-t<-2 g$. Letting $t:=2 s+2 g+1$ we complete the proof.

To construct the required bundle $L$ consider a positive linear vector bundle $E$ on $\mathbf{C T}^{g}$ that determines an imbedding into some linear projective space. Let $\Theta(E) \in \Omega^{1,1}\left(\mathbf{C T}^{g}\right)$ be the curvature form of $E$. Now observe that the function $\log (\rho)$ satisfies

(i) $d \log (\rho)$ is bounded on $\mathbf{C}^{g}$ with respect to the Euclidean metric; 
(ii) the Levi form $\mathcal{L}(\log (\rho))$ is bounded on $\mathbf{C}^{g}$ with respect to the Euclidean metric.

Then there exists a postive integer $n:=n(l, E)$ such that for the linear vector bundle $L:=E^{\otimes n}$ the inequality

$$
\Theta(L)=n \Theta(E) \geq(2(g !) l+2 g+1) \mathcal{L}(\log (\rho))+\epsilon \omega
$$

holds on $\mathbf{C}^{g}$ for some $\epsilon>0$.

Let now $f \in \mathcal{P}_{s}\left(C_{G}\right)$ and $s \leq(g !) l$. By Lemma $4.2 f$ belongs to the weighted space $L_{2}\left(w, C_{G}\right)$ with the weight $w=\exp (-(2 s+2 g+1) \log (\rho))$. This and inequality (4.2) show that the conditions of Lárusson's extension theorem (see [La], th.3.1) are fulfilled for $f$ and zero loci $C$ of $L$. Applying his result we can extend $f$ to a holomorphic function $f^{\prime} \in L_{2}(\exp (-(2 s+$ $\left.2 g+1) \log (\rho)), \mathbf{C}^{g}\right)$. By subharmonity of $\left|f^{\prime}\right|$ from here it follows (see, e.g., [FN], p.1117) that $f^{\prime}$ is a holomorphic polynomial of degree at most $s$. So, $f$ is the restriction of the polynomial $f^{\prime}$ to $C_{G}$.

Going over to the covering $M_{G}$ over the curve $M$ consider the direct and the symmetric products $M^{\times g}$ and $S M^{\times g}$ of $g$-copies of $M$. Then $S M^{\times g}$ is the quotient manifold of $M^{\times g}$ under the action of the permutation group $S_{g}$. Therefore there exists the finite holomorphic surjective mapping $M^{\times g} \longrightarrow$ $S M^{\times g}$. Further, $S M^{\times g}$ is birational isomorphic to $\mathbf{C T}^{g}$ (see, e.g., [GH]). Denote by $j: M^{\times g} \longrightarrow \mathbf{C T}^{g}$ the composition of these two mappings. Let $X \subset M^{\times g}$ be an irreducible component of $j^{-1}(C)$, which without loss of generality we can assume to be a smooth curve. $X$ clearly satisfies the following conditions:

(i) $\left.j\right|_{X}$ is a finite branched covering over $C$ of degree at most $\left|S_{g}\right|=g$ !;

(ii) there exists a holomorphic surjective mapping $h: X \longrightarrow M$ (restriction to $X$ of projection on a factor of $M^{\times g}$ ).

Let $G^{\prime}$ be the image of $\pi_{1}(X)$ under the composite homomorphism $\pi_{1}(X) \stackrel{j_{*}}{\longrightarrow} \pi_{1}(C) \longrightarrow \pi_{1}\left(\mathbf{C T}^{g}\right)$. Clearly $G^{\prime}$ is of a finite index in $\pi_{1}\left(\mathbf{C T}^{g}\right)$. Consider the regular covering $X_{G^{\prime}}$ over $X$ with the transformation group $G^{\prime}$.

Lemma 4.3. There exist finite branched coverings $j^{\prime}: X_{G^{\prime}} \longrightarrow C_{G}$ and $h^{\prime}: X_{G^{\prime}} \longrightarrow M_{G}$ such that

(i) $j^{\prime}$ and $h^{\prime}$ are equivariant with respect to the action of $G^{\prime}$ :

$$
j^{\prime}(g z)=g j^{\prime}(z) \quad \text { and } \quad h^{\prime}(g z)=g h^{\prime}(z) \quad\left(g \in G^{\prime}\right) ;
$$


(ii) $j^{\prime}$ is a covering to $j$.

Proof. In what follows we think of $M$ as a subvariety of $\mathbf{C T}^{g}$. Let $\eta_{1}, \ldots, \eta_{g}$ be a basis for the space $\Omega^{1}\left(\mathbf{C T}^{g}\right)$ of holomorphic 1-forms on $\mathbf{C T}^{g}$. Consider $g$ dimensional subspaces $E_{j}$ and $E_{h}$ of $\Omega^{1}(X)$ spanned by $(j)^{*}\left(\eta_{1}\right), \ldots,(j)^{*}\left(\eta_{g}\right)$ and $(h)^{*}\left(\eta_{1}\right), \ldots,(h)^{*}\left(\eta_{g}\right)$, respectively. Denote by $F_{j}$ and $F_{h}$ their complements in $\Omega^{1}(X)$. Then there exists a C-linear isomorphism $\phi: \Omega^{1}(X) \longrightarrow$ $\Omega^{1}(X)$ such that

$$
\phi\left(F_{h}\right)=F_{j}, \quad \phi\left((h)^{*}\left(\eta_{i}\right)\right):=(j)^{*}\left(\eta_{i}\right), \quad(1 \leq i \leq g) .
$$

If $\omega_{1}, \ldots, \omega_{s}$ is a basis of $F_{h}$, then $\phi\left(\omega_{1}\right), \ldots, \phi\left(\omega_{s}\right)$ is a basis of $F_{j}$. Define now two lattices $\Gamma_{j}$ and $\Gamma_{h}$ of $\mathbf{C}^{g+s}$ by

$$
\begin{aligned}
\Gamma_{j} & :=\left\{g=\left(\int_{\gamma} j^{*}\left(\eta_{1}\right), \ldots, \int_{\gamma} j^{*}\left(\eta_{g}\right), \int_{\gamma} \phi\left(\omega_{1}\right), \ldots, \int_{\gamma} \phi\left(\omega_{s}\right)\right) ; \gamma \in H_{1}(X, \mathbf{Z})\right\} ; \\
\Gamma_{h} & :=\left\{g=\left(\int_{\gamma} h^{*}\left(\eta_{1}\right), \ldots, \int_{\gamma} h^{*}\left(\eta_{g}\right), \int_{\gamma} \omega_{1}, \ldots, \int_{\gamma} \omega_{s}\right) ; \gamma \in H_{1}(X, \mathbf{Z})\right\} .
\end{aligned}
$$

Let $T_{j}:=\mathbf{C}^{g+s} / \Gamma_{j}$ and $T_{h}:=\mathbf{C}^{g+s} / \Gamma_{h}$ be the corresponding complex tori. The Albanese mappings constructed by the above two bases of $\Omega^{1}(X)$ imbed $X$ into $T_{j}$ and $T_{h}$, respectively. Denote the corresponding image of $X$ in $T_{j}$ by $X_{j}$ and in $T_{h}$ by $X_{h}$. In virtue of (4.3) the linear operator $\phi$ induces an isomorphism of $\Omega^{1}\left(T_{h}\right)$ onto $\Omega^{1}\left(T_{j}\right)$. Since the latter two spaces are naturally isomorphic to $\mathbf{C}^{g+s}$, we determine in this way the $\mathbf{C}$-linear automorphism $\phi^{\prime}$ of $\mathbf{C}^{g+s}$. Let $I: \mathbf{C}^{g+s} \longrightarrow \mathbf{C}^{g+s}$ be the $\mathbf{C}$-linear operator conjugate to $\phi^{\prime}$. Then $I: \Gamma_{j} \longrightarrow \Gamma_{h}$ and therefore $I$ determines the biholomorphic mapping $I^{\prime}$ which maps $T_{j}$ onto $T_{h}$. Moreover, $I^{\prime}$ maps $X_{j}$ onto $X_{h}$. Consider the induced homomorphisms $j_{*}: \pi_{1}\left(X_{j}\right) \longrightarrow \pi_{1}\left(\mathbf{C T}^{g}\right)$ and $\left(h \circ I^{\prime}\right)_{*}: \pi_{1}\left(X_{j}\right) \longrightarrow$ $\pi_{1}\left(\mathbf{C T}^{g}\right)$ and show that

$$
\operatorname{Ker}\left(j_{*}\right)=\operatorname{Ker}\left(\left(h \circ I^{\prime}\right)_{*}\right) .
$$

Note that $h \in \operatorname{Ker}\left(j_{*}\right)$ if and only if

$$
\int_{j(h)} \eta_{i}=0 \quad(1 \leq i \leq g)
$$

and $l \in \operatorname{Ker}\left(\left(h \circ I^{\prime}\right)_{*}\right)$ if and only if

$$
\int_{\left(h \circ I^{\prime}\right)(l)} \eta_{i}=0 \quad(1 \leq i \leq g) .
$$


So for such $l$ we have

$$
0=\int_{\left(h \circ I^{\prime}\right)(l)} \eta_{i}=\int_{I^{\prime}(l)} h^{*}\left(\eta_{i}\right)=\int_{l} j^{*}\left(\eta_{i}\right)=\int_{j(l)} \eta_{i}
$$

and hence $l \in \operatorname{Ker}\left(j_{*}\right)$. The inverse imbedding is proved similarly.

Since $j\left(X_{j}\right)=C$ and $\left(h \circ I^{\prime}\right)\left(X_{j}\right)=M$, equality (4.4) leads to existence of holomorphic mappings $j^{\prime}: X_{G^{\prime}} \longrightarrow C_{G}$ and $h^{\prime}: X_{G^{\prime}} \longrightarrow M_{G}$ that cover $j$ and $h \circ I^{\prime}$, respectively. These mappings clearly satisfy the statement of the lemma.

We are now in a position to prove the theorem for the complex curve $M$. By Propositions 4.1 and $3.2 \mathcal{P}_{l}\left(X_{G^{\prime}}\right)$ is finite dimensional. Moreover, by statement $(i)$ of the previous lemma $\left(h^{\prime}\right)^{*}$ determines imbedding of $\mathcal{P}_{l}\left(M_{G}\right)$ into $\mathcal{P}_{l}\left(X_{G^{\prime}}\right)$. Therefore $\mathcal{P}_{l}\left(M_{G}\right)$ is finite dimensional, as well. Apply now to our situation the result of Theorem 1.1. The functions $f_{i}$ of the theorem in this case coincide with the pullback of linear holomorphic functions defined on $\mathbf{C}^{g}$ by the mapping $A^{\prime}: M_{G} \longrightarrow \mathbf{C}^{g}$ which covers the Albanese mapping $A$. From here it follows that every $f \in \mathcal{P}_{l}\left(M_{G}\right)$ is the pullback of a holomorphic polynomial of degree at most $l$ on $\mathbf{C}^{g}$.

This completes the proof in the case of curves.

Let now $M \subset \mathbf{C P}^{n}$ be a projective manifold. Since the Albanese mapping from $M$ into its Picard manifold (complex torus) induces a surjective homomorphism of $\pi_{1}(M)$ onto the maximal free abelian subgroup $G$ of $H_{1}(M, \mathbf{Z})$, it suffices to prove the theorem for the covering $M_{G}$. By Bertini's theorem the generic subspace $L$ of codimension $\operatorname{dim}_{\mathbf{C}} M-1$ intersects $M$ transversely in a smooth curve $C$ and the imbedding $C \subset M$ induces the surjective homomorphism of fundamental groups. Moreover, the union $U$ of such curves $C$ is an open subset of $M$. Finally, according to Theorem 1.2 in the case of curves $C_{G} \in \mathcal{P F}$. So the conditions of Proposition 3.1 are fulfilled for the family of curves $C$ and therefore $M_{G} \in \mathcal{P F}$, that is, the theorem is proved in this case, as well.

Finally, consider the general case of a compact complex manifold $M$ and a holomorphic mapping $A: M \longrightarrow \mathbf{C T}^{r}$ which induces the surjective homomorphism of the corresponding fundamental groups. Show that this case can be reduced to the case of a manifold $Y^{\prime}$ which is a desingularisation $d_{Y}: Y^{\prime} \longrightarrow Y$ of the image $Y:=A(M)$. To accomplish this note that there exist a desingularisation $d_{M}: M^{\prime} \longrightarrow M$ and a holomorphic surjective mapping $B: M^{\prime} \longrightarrow Y^{\prime}$ such that

$$
d_{Y} \circ B=A \circ d_{M} .
$$


It is clear that $M^{\prime}$ is birational equivalent to $M$ and therefore $\pi_{1}\left(M^{\prime}\right) \cong$ $\pi_{1}(M)$. Consider regular coverings $M_{G}^{\prime}$ and $M_{G}$ with the transformation group $G:=A_{*}\left(\pi_{1}(M)\right)$ over $M^{\prime}$ and $M$, respectively. Then there exists covering to $d_{M}$ holomorphic mapping $h: M_{G}^{\prime} \longrightarrow M_{G}$ which is a birational isomorphism too. From here it follows that $(h)^{*}: \mathcal{P}_{l}\left(M_{G}\right) \longrightarrow \mathcal{P}_{l}\left(M_{G}^{\prime}\right)$ is an isomorphism. Thus it suffices to prove the theorem for $M_{G}^{\prime}$.

Let now $\left(V, f_{1}, f_{2}\right)$ be the Stein factorization of $B: M^{\prime} \longrightarrow Y^{\prime}$, that is, $V$ be a compact complex variety, $f_{1}: M^{\prime} \longrightarrow V$ be surjective with connected fibers and $f_{2}: V \longrightarrow Y^{\prime}$ be a finite branched covering such that $B=f_{2} \circ f_{1}$. Because of surjectivity of $A_{*}$ one can define correctly coverings $V_{G}$ and $Y_{G}^{\prime}$ with the same transformation group $G$ over $V$ and $Y^{\prime}$, respectively. Then there exist covering to $f_{1}$ and $f_{2}$ mappings $f_{1}^{\prime}$ : $M_{G}^{\prime} \longrightarrow V_{G}$ and $f_{2}^{\prime}: V_{G} \longrightarrow Y_{G}^{\prime}$ equivariant with respect to the actions of $G$. Every fiber of $f_{1}^{\prime}$ is a connected compact complex variety and therefore $\left(f_{1}^{\prime}\right)^{*}$ determines an isomorphism of $\mathcal{P}_{l}\left(V_{G}\right)$ onto $\mathcal{P}_{l}\left(M_{G}^{\prime}\right)$. So it remains to proof that $V_{G} \in \mathcal{P F}$. But we can apply Proposition 3.2 (see also Remark 3.3) to the case of the finite branched covering $f_{2}: V \longrightarrow Y^{\prime}$. According to this proposition $V_{G} \in \mathcal{P F}$ if and only if $Y_{G}^{\prime} \in \mathcal{P F}$. So we reduce the problem to the case of a desingularisation $Y^{\prime}$ of $Y=A(M)$. To choose the required desingularisation we make use of the next statement which follows directly from theorem 10.9 of [U].

There exists a desingularisation $Y^{\prime}$ of $Y$, a projective manifold $Z$ and a holomorphic surjective mapping $g: Y^{\prime} \longrightarrow Z$ such that $g$ has connected fibers and the generic fiber of $g$ is biholomorphic to a complex torus.

Taking the $Y^{\prime}$ from the statement and applying Theorem 1.2 to the case of projective manifolds and complex tori and then applying Proposition 3.3 to the mapping $g$ we obtain that $Y_{G}^{\prime} \in \mathcal{P F}$.

This completes the proof of the theorem.

\section{Proof of Corollaries.}

Proof of Corollary 1.3. Let $M_{G}$ be a regular covering over a compact Kähler manifold $M$ with a free abelian transformation group $G$. Since $\mathcal{P}_{l}\left(M_{G}\right)$ is linearly imbedded into $\mathcal{P}_{l}\left(M_{F}\right)$, where $F$ is the maximal free abelian subgroup of $H_{1}(M, \mathbf{Z})$ it suffices to consider the case $G=F$. The Albanese mapping $A: M \longrightarrow \mathbf{C T}^{r}$, where $r:=\frac{1}{2} \operatorname{dim}_{\mathbf{C}} H_{1}(M, \mathbf{C})$ induces the surjective homomorphism $A_{*}$ onto $F$. So Theorem 1.2 with this $A$ imposes that 
every $f \in \mathcal{P}_{l}\left(M_{G}\right)$ has a form $\left(A^{\prime}\right)^{*}(p)$. Here $A^{\prime}: M_{G} \longrightarrow \mathbf{C}^{r}$ is the covering of $A$ and $p$ is a holomorphic polynomial on $\mathbf{C}^{r}$ of degree at most $l$.

Proof of Corollary 1.4. Let $M_{G}$ be a regular covering over a compact complex manifold $M$ with $\tau(M) \geq \operatorname{dim}_{\mathbf{C}} M-1$ and a free abelian transformation group $G$. Recall that $\tau(M)$ is the transcendency degree of the field of meromorphic functions on $M$. As above it suffices to consider the case where $G$ is the maximal free abelian subgroup of $H_{1}(M, \mathbf{Z})$. First consider the case $\tau(M)=\operatorname{dim}_{\mathrm{C}} M$. Then $M$ is birational equivalent to a compact projective manifold $M^{\prime}$ (see, e.g., [Sh], ch.8, sec.3) and, in particular, $\pi_{1}(M) \cong \pi_{1}\left(M^{\prime}\right)$. Applying Corollary 1.3 to the projective manifold $M^{\prime}$ we get the required result.

Let now $\tau(M)=\operatorname{dim}_{\mathrm{C}} M-1$. Then there exists a modification $p$ : $M^{\prime} \longrightarrow M$ such that $M^{\prime}$ admits a holomorphic surjective mapping onto a projective manifold of complex dimension $\operatorname{dim}_{\mathrm{C}} M-1$ and the generic fiber of this mapping is biholomorphic to an elliptic curve (see, e.g., [Sh], ch.8, sec.4). It remains to apply Theorem 1.2 in the case of projective manifolds and elliptic curves and then to use Proposition 3.3.

Proof of Corollary 1.5. Let $M_{G}$ be a regular covering over a compact complex curve $M$ of genus $g$. Here $G$ is a free abelian transformation group which without loss of generality to be assumed maximal. We have to prove that every $f \in \mathcal{H}_{l}\left(M_{G}\right)$ can be represented as $\left(A^{\prime}\right)^{*}(p)$ with a harmonic polynomial $p$ on $\mathbf{C}^{g}$ which is a sum of holomorphic and antiholomorphic ones of degrees at most $l$. Here $A^{\prime}: M_{G} \longrightarrow \mathbf{C}^{g}$ is the covering of the Albanese mapping $A: M \longrightarrow \mathbf{C T}^{g}$. In what follows we regard $M$ as a submanifold of $\mathbf{C T}^{g}$. Since $\mathbf{C T}^{g}$ is a complex abelian Lie group, there exists a neighborhood $U \subset \mathbf{C T}^{g}$ of $M$ such that $M$ is a holomorphic retract of $U$. Let $U_{G}$ be the preimage of $U$ in $\mathbf{C}^{g}$ with respect to the canonical covering $\mathbf{C}^{g} \longrightarrow \mathbf{C T}^{g}$ and $r: U_{G} \longrightarrow M_{G}$ be the corresponding retraction. Since $f$ is a harmonic function on the complex curve $M_{G}$, it satisfies the equation $\partial \bar{\partial} f=0$. In particular, $f$ is locally a sum of holomorphic and antiholomorphic functions. Because of holomorphicity of $r, \partial \bar{\partial}\left(r^{*} f\right)=0$. In addition, $r^{*} f$ is an $l$-growth function on $U_{G}$ with respect to the Euclidean metric on $\mathbf{C}^{g}$. Then the Cauchy inequalities for derivatives of the holomorphic part of $r^{*} f$ show that 
the $d$-closed holomorphic 1-form $\alpha:=\partial\left(r^{*} f\right)$ can be represented as follows:

$$
\alpha=\sum_{i=1}^{g} a_{i}(z) d z_{i}
$$

where $a_{i}$ is a holomorphic function of $l$-growth on $U_{G}, 1 \leq i \leq g$. Theorem 1.2 implies that $\left.a_{i}\right|_{M_{G}}$ is restriction to $M_{G}$ of a holomorphic polynomial of degree at most $l$. Therefore $\Delta_{g_{1}, \ldots, g_{l+1}}\left(a_{i}\right)=0$, where $g_{1}, \ldots, g_{l+1}$ belong to $G$. Since $f=\left.r^{*} f\right|_{M_{G}}$ we get from here that $\partial\left(\Delta_{g_{1}, \ldots, g_{l+1}} f\right)=0$. The similar arguments applied to $\bar{\partial}\left(r^{*} f\right)$ lead to the equality $\bar{\partial}\left(\Delta_{g_{1}, \ldots, g_{l+1}} f\right)=0$. These two equalities imply $\Delta_{g_{1}, \ldots, g_{l+1}} f$ is a constant on $M_{G}$ and so every $f \in \mathcal{H}_{l}\left(M_{G}\right)$ is annihilated by any $(l+2)$-difference. Applying the arguments of the proof of Lemma 2.3 we conclude from here that $\mathcal{H}_{l}\left(M_{G}\right)$ is finite dimensional. In addition, the induction arguments of Theorem 1.1 show that every $f \in \mathcal{H}_{l}\left(M_{G}\right)$ can be written as $f=\left(A^{\prime}\right)^{*}(p)$ with a polynomial $p$ on $\mathbf{C}^{g}$ of degree at most $l$.

It remains to prove that the $p$ is sum of holomorphic and antiholomorphic polynomials. To this end note that the restrictions $\left.a_{i}\right|_{M_{G}}$ of the coefficients of the above defined holomorphic 1-form $\alpha$ coincide with restrictions to $M_{G}$ of holomorphic polynomials on $\mathbf{C}^{g}$. So we can extend $\left.\alpha\right|_{M_{G}}$ to a holomorphic polynomial 1-form $\beta$ and therefore the polynomial $p$ satisfies

$$
\partial p-\beta=0 \text { on } M_{G} \text {. }
$$

Check that $\partial p-\beta=\sum_{i=1}^{g} p_{i}(z) d z_{i}$ equals 0 identically on $\mathbf{C}^{g}$. Assume to the contrary that $p_{i_{0}} \neq 0$ for some $i_{0}$. Then there exists a difference operator $\Delta_{g_{1}, \ldots, g_{t}}$ such that $\Delta_{g_{1}, \ldots, g_{t}}(\partial p-\beta) \neq 0$ but $\Delta_{g}\left(\Delta_{g_{1}, \ldots, g_{t}}(\partial p-\beta)\right)=0$ for any $g \in G$. From here we get

$$
\Delta_{g_{1}, \ldots, g_{t}}(\partial p-\beta)=\sum_{i=1}^{g} c_{i} d z_{i}
$$

with some constants $c_{i}$. But $\left.(\partial p-\beta)\right|_{M_{G}}=0$ and the forms $\left.d z_{1}\right|_{M_{G}}$ $, \ldots,\left.d z_{g}\right|_{M_{G}}$ are linearly independent. Hence all $c_{i}=0$ and we get a contradiction to the condition $\Delta_{g_{1}, \ldots, g_{t}}(\partial p-\beta) \neq 0$.

Thus $\partial p-\beta=0$ and, in particular, $\partial p$ is a $d$-closed holomorphic 1-form on $\mathbf{C}^{g}$. Therefore the holomorphic function

$$
h_{1}:=\int_{z_{0}}^{z} \partial p
$$


is correctly defined on $\mathbf{C}^{g}$. Moreover, the growth conditions on $p$ show that $h_{1}$ is a holomorphic polynomial. Then $h_{2}:=p-h_{1}$ is an antiholomorphic polynomial and therefore $p=h_{1}+h_{2}$ is harmonic.

Remark 5.1. The latter corollary can be extended with the same proof to the case of $l$-polynomial growth functions $f$ on $M_{G}$ with a compact Kähler manifold $M$ satisfying $\bar{\partial} \partial f=0$. In the case of curves the latter condition is equivalent to the harmonicity of $f$.

\section{References.}

[AL] M. Avallaneda and F.-H. Lin, Une théorème de Liouville pour des équations elliptiques à coefficients périodiques, Compt. Rendus Acad. Sci. Paris, Sér. I, 309 (1989), 245-250.

[CM] T. Colding and W. Minicozzi, Weyl type bounds for harmonic functions, preprint.

[FN] C. Fefferman and R. Narasimhan, On the polynomial-like behavior of certain algebraic functions, Ann. Inst. Fourier, Grenoble, 44 (1994), 1091-1179.

[G] F.R. Gantmaher, Matrix Theory, "Nauka", Moscow, 1967.

[Gu] Y. Guivarch, Mouvement brownien sur les revêtements d'une variété compacte, C.R. Acad. Sci. Paris, Sér. I, 292 (1981), 851-853.

[GH] Ph. Griffiths and J. Harris, Principles of Algebraic Geometry, WileyInterscience, N.Y., 1978.

[K] V. Kaimanovich, Brownian motion and harmonic functions on covering manifolds. An entropy approach, Soviet Mathematics Doklady, 33 (1986), 812816.

[La] F. Lárusson, An extension theorem for holomorphic functions of slow growth on covering spaces of projective manifolds, J. of Geometric Analysis, 5 (1995), 281-291.

[L] V. Lin, Liouville coverings of complex spaces and amenable groups, Mathematics of the USSR-Sbornik, 60 (1988), 197-216.

[Li] P. Li, Harmonic sections of polynomial growth, Math. Research Letters, 4 (1997), 35-44.

[LS] T. Lyons and D. Sullivan, Function theory, random paths and covering spaces, J. of Differential Geometry, 19 (1984), 299-323. 
[MS] J. Moser and M. Struwe, On a Liouville-type theorem for linear and nonlinear elliptic differential equations on a torus, ETH, Zürich, 1991.

[OV] A. Onishchik and E. Vinberg, Seminar in Lie groups and algebraic groups, Springer-Verlag, 1992.

[Sh] I. Shafarevich, Basic Algebraic Geometry 2. Schemes and complex manifolds, Springer-Verlag, 1994.

[U] K. Ueno, Classification theory of algebraic varieties and compact complex surfaces, Lecture Notes in Math. 439, Springer-Verlag, 1975.

[Y] S.T. Yau, Nonlinear Analysis in Geometry, L'Enseignement Mathématique, Série des Conférences de l'Union Mathématique Internationale, No. 8, SROKUNDIG, Genève, 1986.

Received September 10, 1996.

DePaRTMENT OF Mathematics

UNIVERSITY OF TORONTO

100 St. GEorge St.

TORONTO, ON M5S 1A1

CANADA

E-MAIL: BRUDNYI@MAS.TORONTO.EDU 